\title{
Alcohol and other psychoactive drugs in trauma patients aged $10-14$ years
}

\author{
Guohua Li, Arjun Chanmugam, Richard Rothman, Carla DiScala, Charles N Paidas, \\ Gabor D Kelen
}

\begin{abstract}
Objective-To examine the prevalence of alcohol and/or other psychoactive drugs, such as marijuana and cocaine (AODs), involved in preteen trauma patients.

Methods-Toxicological testing results were analyzed for 1356 trauma patients aged 10-14 years recorded in the National Pediatric Trauma Registry for the years 1990-95.

Results-Of the 1356 patients who received toxicological screening at the time of admission, $116(9 \%)$ were positive for AODs. AOD involvement increased with age. Patients with pre-existing mental disorders were nearly three times as likely as other patients to be AOD positive (23\% $v 8 \%, \mathrm{p}<0.01)$. AOD involvement was more prevalent in intentional injuries and in injuries that occurred at home.

Conclusions-AODs in preteen trauma are of valid concern, in particular among patients with mental disorders or intentional injuries. The role of AODs in childhood injuries needs to be further examined using standard screening instruments and representative study samples.

(Injury Prevention 1999;5:94-97)
\end{abstract}

Keywords: alcohol; substance abuse; trauma

The past decade has witnessed great strides in understanding the relationship between alcohol and trauma. In addition to further documenting the role of acute alcohol use in various types of injuries, researchers have probed the interplay of chronic problem drinking with trauma. It is well recognized that alcoholism is prevalent, not only among trauma patients with raised blood alcohol concentrations (BACs), but also among those who are negative for alcohol at the time of admission. ${ }^{1-3}$ Research also indicates that intoxicated trauma patients are significantly more likely than the sober to be admitted for repeat injury, ${ }^{45}$ and that trauma patients with chronic alcohol abuse are at increased risk for injury complications, such as pulmonary infection. ${ }^{6}$ Because of the clinical implications of alcohol abuse for trauma management, alcohol testing has become routine in many adult trauma centers, and screening for alcoholism and providing drug abuse treatment are increasingly integrated into trauma care programs. ${ }^{37}$

These developments, however, are limited to adults. A few studies involving adolescent trauma patients indicate that more than one third have positive BACs at admission. ${ }^{8-10}$ Few studies have examined the role of alcohol and/or other psychoactive drugs (AODs) in pediatric trauma. The purposes of this study were to document the prevalence of AODs in trauma patients aged 10-14 years and to examine demographic characteristics and injury circumstances associated with AOD involvement among these patients.

\section{Methods}

The National Pediatric Trauma Registry (NPTR) is a multi-institutional data system designed to collect information for studying acute care management and functional outcomes of injured children and adolescents. Started in 1985, the NPTR has evolved into a valuable data source for clinical research on the epidemiology, treatment, rehabilitation, and prevention of pediatric trauma. ${ }^{11-17}$ As of December 1995, there were 76 hospitals (74 in the continental United States, one in Puerto Rico, and one in Canada) voluntarily participating in this registry. Using standard forms and procedures, each participating hospital is required to submit data on all patients under 20 years of age who were admitted with injury as the primary diagnosis (except poisoning, burns, and near drowning). These data are sent to the registry at New England Medical Center, Boston, Massachusetts.

Data collected include information on demographics, injury circumstances, pre-hospital and post-admission trauma management, and assessment of functional status at discharge. To assure data accuracy and completeness, each record is reviewed by a staff member before being entered into the database. Logic checking is conducted automatically by specially designed computer programs. If illogical or missing data are found, the submitting hospital is requested to provide supplemental information for correction or clarification. The methods of data collection and quality assur-

Street, Baltimore, $M$

21287-2080, USA (e-mail: ghli@jhmi.edu). 
Table 1 Prevalence of AODs detected among 1356 trauma patients aged 10-14 years, NPTR, 1990-95

\begin{tabular}{lcl}
\hline & No of patients & Prevalence (\%) \\
\hline Alcohol & 24 & 1.8 \\
Non-alcohol drugs & 94 & 6.9 \\
Cocaine & 17 & 1.3 \\
Opiate & 15 & 1.1 \\
Amphetamine & 3 & 0.2 \\
Marijuana & 32 & 2.4 \\
Benzodiazepine & 24 & 1.8 \\
Other & 3 & 0.2 \\
\hline
\end{tabular}

^For patients who were positive for multiple drugs, only the most potent drug was counted.

ance used in the NPTR are described in detail elsewhere. ${ }^{11}$

This study included all patients aged 10-14 years who were admitted to the participating hospitals of the NPTR from 1 January 1990 to 31 December 1995 and for whom a toxicological screening was performed at admission. Although trauma care protocols recommend that toxicological screening for AODs be performed for all teenagers and adults, in practice the testing is often done based on the type of injury and suspicion of usage, especially for alcohol. The NPTR contains several data items indicating whether the toxicological screening was done; if it was done, whether the result was negative or positive; if positive for alcohol, the BAC was recorded; and if positive for other drugs, the substance names were listed. BAC was assessed by serum and other drugs by urinalysis. In this study, patients who tested positive only for non-illicit drugs (for example, caffeine and nicotine) or prescribed medications (for example, antibiotics) were coded as AOD negative, along with patients whose toxicological screening revealed no drugs at all. Patients who tested positive for either alcohol or other psychoactive drugs were classified as AOD positive. Included in psychoactive drugs were opiate and benzodiazepine, though they could

Table 2 Factors related to the presence of AODs in trauma patients aged 10-14 years, NPTR, 1990-95

\begin{tabular}{|c|c|c|c|c|}
\hline \multirow[b]{2}{*}{ Variable } & \multirow{2}{*}{$\begin{array}{l}\text { No (\%) } A O D \\
\text { positive }\end{array}$} & \multirow{2}{*}{$\begin{array}{l}\text { No (\%) AOD } \\
\text { negative }\end{array}$} & \multicolumn{2}{|c|}{ Odds ratio } \\
\hline & & & Crude & Adjusted \\
\hline \multicolumn{5}{|l|}{ Age (years) } \\
\hline $10-13$ & $52(6.1)$ & 807 (93.9) & 1.0 & 1.0 \\
\hline 14 & $64(12.9)$ & $433(87.1)$ & $2.3 \dagger$ & $2.1 \dagger$ \\
\hline \multicolumn{5}{|l|}{ Sex } \\
\hline Female & $29(6.8)$ & $398(93.2)$ & 1.0 & 1.0 \\
\hline Male & $87(9.4)$ & $842(90.6)$ & 1.4 & 1.3 \\
\hline \multicolumn{5}{|l|}{ Pre-existing mental disorder } \\
\hline No & $97(7.6)$ & $1176(92.4)$ & 1.0 & 1.0 \\
\hline Yes & $19(22.9)$ & $64(77.1)$ & $3.6+$ & $2.9 \dagger$ \\
\hline \multicolumn{5}{|l|}{ Day of injury } \\
\hline Monday to Friday & $73(7.8)$ & $857(92.2)$ & 1.0 & 1.0 \\
\hline Saturday to Sunday & $43(10.1)$ & $383(89.9)$ & 1.3 & 1.4 \\
\hline \multicolumn{5}{|l|}{ Time of injury ${ }^{\star}$} \\
\hline $6: 00$ am to $5: 59 \mathrm{pm}$ & $47(7.7)$ & $565(92.3)$ & 1.0 & - \\
\hline $6: 00 \mathrm{pm}$ to $5: 59 \mathrm{am}$ & $53(10.3)$ & $462(89.7)$ & 1.4 & - \\
\hline \multicolumn{5}{|l|}{ Mechanism of injury } \\
\hline Traffic & $47(6.5)$ & $675(93.5)$ & 1.0 & 1.0 \\
\hline Non-traffic unintentional & $29(7.4)$ & $362(92.6)$ & 1.2 & 1.0 \\
\hline Self inflicted & $9(27.3)$ & $24(72.7)$ & $5.4 \dagger$ & 2.6 \\
\hline Assault & $31(14.8)$ & $179(85.2)$ & $2.5 \dagger$ & $2.4 \ddagger$ \\
\hline \multicolumn{5}{|l|}{ Scene of injury } \\
\hline Road & $58(7.1)$ & $758(92.9)$ & 1.0 & 1.0 \\
\hline Home & $28(13.8)$ & $175(86.2)$ & $2.1 \dagger$ & 1.5 \\
\hline Other & $30(8.9)$ & $307(91.1)$ & 1.3 & 0.9 \\
\hline
\end{tabular}

*Time of injury was unknown for 229 patients and was not included in the multivariate logistic regression model because of the large proportion of missing data.

+ Significant at $\mathrm{p}<0.01$ level.

$\ddagger$ Significant at $\mathrm{p}<0.05$ level. come from sources of non-abusive use. AOD involvement was examined in relation to patient characteristics and injury circumstances. Variables examined in this study include patient age, gender, pre-existing mental disorder, time of injury, day of injury (weekday or weekend), mechanism of injury (traffic, unintentional other than traffic, self inflicted, or assault), and scene of injury (home, road, or other public place such as school, park, and sports field). Information about socioeconomic status was not available from the NPTR. Pre-existing medical conditions, including mental disorders, were those that existed before the injury and were recorded by clinicians at admission based on self or proxy reports.

Data were analyzed using the Statistical Analysis System (SAS) software (SAS Institute, Cary, NC). The association of patient characteristics and injury circumstances with AOD involvement was assessed based on both crude and adjusted odds ratios.

\section{Results}

During the six year study period, the NPTR recorded 11357 trauma patients aged 10-14 years. Toxicological screening was conducted for $1356(12 \%)$ of these patients. Patients who received toxicological screening were significantly more likely than those who did not to be injured at night (6:00 pm to 5:59 am) (46\% v $40 \%$ ), to be victims of assault or self inflicted injury $(15 \% v 5 \%)$, and to have pre-existing mental disorder $(6 \%$ v $3 \%)$. Toxicological screening was not associated with patient gender or day of the week. During the study period, the proportion of patients who received toxicological screening decreased from $15 \%$ in 1990 to $9 \%$ in 1995 .

Of the 1356 patients who received toxicological screening, $24(1.8 \%)$ tested positive for alcohol (table 1), with BACs ranging from $7-230 \mathrm{mg} / \mathrm{dl}$. Among positive BACs, $68 \%$ were $100 \mathrm{mg} / \mathrm{dl}$ or higher. The mean BAC for the alcohol positive patients was $138 \mathrm{mg} / \mathrm{dl}$, similar between boys $(139 \mathrm{mg} / \mathrm{dl})$ and girls (136 $\mathrm{mg} / \mathrm{dl}$ ). Among the 24 alcohol positive patients, only two were also positive for cocaine.

Other psychoactive drugs were found in 94 $(6.9 \%)$ of the patients (table 1$)$. The most common drug detected was marijuana, followed by benzodiazepine, cocaine, and opiate (table 1). Among the 94 patients who tested positive, $24(26 \%)$ used multiple drugs.

Together, AODs were found in $116(9 \%)$ of the patients. The proportion of patients who were AOD positive varied with demographic characteristics and injury circumstances (table 2 ). Of the patients aged 14, 13\% were AOD positive, compared with $6 \%$ among younger patients $(p<0.01)$. Before injury, $6 \%$ of the patients had mental disorders, such as disturbance of conduct and depression. Patients who were reported having pre-existing mental disorder were three times as likely as other patients to be AOD positive $(22.9 \%$ v $7.6 \%$, $\mathrm{p}<0.01)$. AOD involvement was also associated with both the mechanism and the scene of 


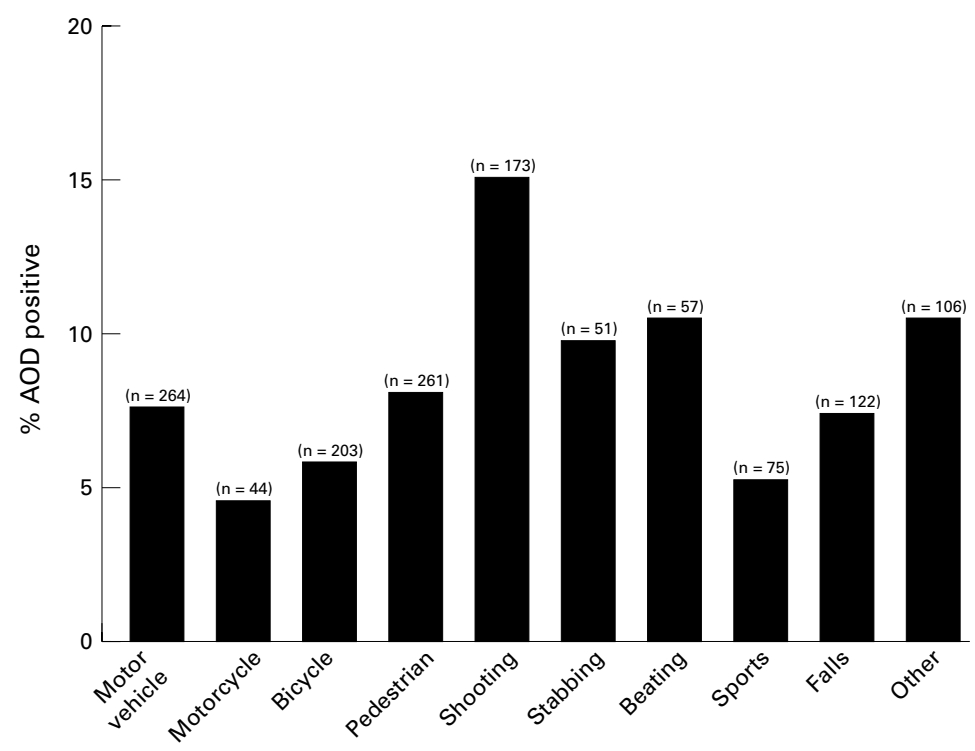

Figure 1 Percentages of trauma patients aged 10-14 years who were AOD positive by mechanism of injury, NPTR, 1990 to 1995.

injury. Intentional injury and injury that occurred at home were more likely to involve AODs. Among patients with intentional injury, those who were shot were most likely to be AOD positive (15\%), followed by victims of beating (11\%), and stabbing (10\%) (fig 1).

The association of AODs with demographic characteristics and injury circumstances was further assessed using the multivariate logistic regression model (table 2). With adjustment for age, gender, and variables indicating injury circumstances, the odds of being AOD positive for patients with pre-existing mental disorder was 2.9 times that for patients without pre-existing mental disorder. Other factors that were significantly associated with the likelihood of being AOD positive were age and mechanism of injury (table 2).

To evaluate the effect of incomplete testing rate on the estimates of AOD involvement, stratification analysis was conducted on different hospitals according to the percentage of patients having received toxicological screening. Most of the hospitals tested fewer than $10 \%$ of their patients for AODs. The proportion of patients who were AOD positive decreased from $18 \%$ to $6 \%$ as the testing rate increased from $<10 \%$ to $29 \%$, and became fairly stable after the testing rate reached $20 \%$.

\section{Discussion}

Use of AODs by children has increased in recent years. ${ }^{18}{ }^{19}$ Although it has been reported that alcohol is prevalent in adolescent trauma, the role of AODs in child injuries is largely unknown. Many factors may have contributed to the inadequacy of research on AODs in pediatric trauma, including ethical issues in recruiting child patients and the failure of pediatric trauma care providers to address the psychosocial and behavioral aspects of injury. Results of the present study indicate that about $9 \%$ of the patients aged $10-14$ years who were admitted to trauma centers and received toxicological screening were AOD positive. The prevalence rate of alcohol intoxication in the pediatric trauma population is much lower than reported in adolescent trauma patients. ${ }^{8-10}$ However, the mean BAC for pediatric trauma patients who were alcohol positive was quite high $(138 \mathrm{mg} / \mathrm{dl})$, and in fact similar to that found among adolescents aged 16-20 years. ${ }^{8}$ The high BACs imply that alcohol may have played a significant part in the causality of the injury among the alcohol positive patients. Thus, these patients are at great risk for becoming chronic alcohol abusers and for being injured again. Studies conducted in adult trauma patients revealed that those with substance abuse problems are more than twice as likely as other trauma patients to be hospitalized for repeat injuries. ${ }^{4} 59$

The association of mental disorder with the risk of injury has been suggested in the literature. ${ }^{20}$ Although the present study cannot establish a causal relationship between mental disorder and injury, these data provide more evidence for the association of acute substance abuse with pre-existing mental disorders. The results indicate that $6 \%$ of the preteen trauma patients have pre-existing mental disorders, and those with those disorders are about three times as likely as other patients to be AOD positive at admission. These findings suggest that psychiatric services, including substance abuse counseling and treatment, should be incorporated into the echelons of pediatric trauma care.

The estimated magnitude of AOD involvement in this study is susceptible to selection bias because only $12 \%$ of the patients recorded in the NPTR are screened for AODs and those likely to be intoxicated are more apt to be tested. The percentage of patients who were screened for AODs varied with hospitals. Analysis based on different subgroups of hospitals shows that the estimated prevalence rate of AOD involvement decreased as the testing rate increased. In hospitals with testing rates of $30 \%-76 \%$, the prevalence rate of AOD involvement was $6 \%$, indicating that the magnitude of AOD involvement reported in this study was probably somewhat overestimated. Furthermore, some of the drugs reported in this study, such as benzodiazepine and opiate, could come from medications administered in the emergency department, rather than sources of abusive use. However, the overestimation due to selection bias could be offset by the fact that the time interval from injury to admission was not taken into account in this study, which could result in a false negative for those patients who were admitted several hours after injury. In addition, as toxicological screening could be performed and reported differently across hospitals, some patients might be tested for alcohol only and were recorded as "positive" only if the BAC exceeded the legal limit for drivers.

\section{Implications for prevention}

It is recognized that injury presents a unique opportunity to initiate intervention for substance abuse. ${ }^{71-23}$ Comprehensive instruments 
for assessing alcohol and other drug abuse are readily available and clinical guidelines for referral and treatment have been developed. ${ }^{724}$ Clinical trials conducted in adults demonstrated that brief counseling can reduce alcohol consumption by over $40 \%$ in primary care settings, ${ }^{25} 26$ as well as in inpatient care settings. ${ }^{27}{ }^{28}$ It is unclear, however, whether similar intervention programs can be successfully implemented in children and adolescents. Since alcohol abuse is both an important risk factor for injury and a common comorbidity among trauma patients, it is necessary to apply a holistic approach to preventing injury and substance abuse. Pediatric trauma patients represent a target group of high priority for such intervention programs.

This research was supported in part by a FIRST Award (R29AA099963) from the National Institute on Alcohol Abuse and Alcoholism (GL), by grant R49/CCR302486 from the Centers for Disease Control and Prevention (GDK and GL), and by grant H133850006 from the National Institute on Disability and Rehabilitation Research (CDiS). The authors thank Sophia Sterling, MPH, for data assistance.

1 Soderstrom CA, Dischinger PC, Smith GS, et al. Psychoactive substance dependence among trauma center patients. fAMA 1992;267:2756-9.

2 Rivara FP, Jurkovich GJ, Gurney JG, et al. The magnitude of acute and chronic alcohol abuse in trauma patients. Arch acute and chronic alco

3 Soderstrom CA, Smith GS, Dischinger PC, et al. Psychoactive substance use disorders among seriously injured trauma center patients. $\mathscr{F} A M A 1997 ; 277: 1769-74$

4 Sims DW, Bivins BA, Obeid FN, et al. Urban trauma: a chronic recurrent disease. F Trauma 1989;29:940-6.

5 Rivara FP, Koepsell TD, Jurkovich GJ, et al. The effects of alcohol abuse on readmission for trauma. $\mathcal{F} A M A 1993 ; 270$ 1962-4.

6 Jurkovich GJ, Rivara FP, Gurney JG, et al. The effect of acute alcohol intoxication and chronic alcohol abuse on outcome from trauma. $\mathcal{F A M A} 1993 ; 270$ :51-6.

7 Gentilello LM, Donovan DM, Dunn CW, et al. Alcohol interventions in trauma centers: current practice and future directions. FAMA 1995;274:1043-8.

8 Hicks BA, Morris JA, Bass SM, et al. Alcohol and the adolescent trauma population. $\mathcal{F}$ Pediatr Surg 1990;25:944-9.

9 Maio RF, Portnoy J, Blow FC, et al. Injury type, injury severity, and repeat occurrence of alcohol-related trauma in adolescents. Alcohol Clin Exp Res 1994;18:261-4.
10 Mannenbach MS, Hargarten SW, Phelan MB. Alcohol use among injured patients aged 12-18 years. Acad Emerg Med 997;4:40-4.

11 Tepas JJ, Ramenofsky ML, Barlow B, et al. National Pediatric Trauma Registry. F Pediatr Surg 1989;24:156-8.

12 Osberg JS, DiScala C, Gans BM. Utilization of inpatient rehabilitation services among traumatically injured children discharged from pediatric trauma centers. Am $\mathcal{F}$ Phys Med Rehabil 1990;69:66-72.

13 Osberg JS, DiScala C. Morbidity among pediatric motor vehicle crash victims: the impact of seatbelts. Am f Public Health 1992;82:422-5.

14 Lescohier I, DiScala C. Blunt trauma in children: causes and outcomes of head versus extracranial injury. Pediatrics 1993;91:721-5.

$15 \mathrm{Li} \mathrm{G}$, Baker SP, Fowler C, et al. Factors related to the presence of head injury in bicycle-related pediatric trauma patients. F Trauma 1995;38:871-5.

16 Laraque D, Barlow B, Durkin M, et al. Children who are shot: a 30-year experience. F Pediatr Surg 1995;30:1072-6.

$17 \mathrm{Li} \mathrm{G}$, Baker SP, DiScala C, et al. Factors associated with the intent of firearm-related injuries in pediatric trauma patients. Arch Pediatr Adolesc Med 1996;150:1160-5.

18 Centers for Disease Control and Prevention. Health-risk behaviors among persons aged $12-21$ years, United States, 1992. Morb Mortal Wkly Rep 1994;43:231-5.

19 Centers for Disease Control and Prevention. CDC surveillance summaries. Morb Mortal Wkly Rep 1996;45 (No SS-4):1-84.

20 Larsson JO, Aurelius G. Accidents in childhood: relation to psychosocial conditions and mental development. Acta Paediatr 1996;85:285-91.

21 Maull KI. Alcohol abuse: its implications in trauma care. South Med F 1982;75:794-8.

22 Soderstrom CA, Cowley R. A national alcohol and trauma center survey: missed opportunities, failures of responsibility. Arch Surg 1987;122:1067-71.

23 Center for Substance Abuse Treatment. Alcohol and other drug screening of hospitalized trauma patients. Rockville, MD: Center for Substance Abuse Treatment, 1995. (DHHS publication No (SMA) 95-3041.)

24 Center for Substance Abuse Treatment. Guidelines for the treatment of alcohol- and other drug-abusing adolescents. Rockville, MD: Center for Substance Abuse Treatment, 1993. (DHHS publication No (SMA) 93-2010.

25 Fleming MF, Barry KL, Manwell LB, et al. Brief physician advice for problem alcohol drinkers: a randomized controlled trial in community-based primary care practices. IAMA 1997;277:1039-45.

26 Wallace P, Cutler S, Haines A. Randomised controlled trial of general practioner intervention in patients with excessive alcohol consumption. BMF 1988;297:663-8.

27 Chick J, Lloyd G, Crombie E. Counselling problem drinkers in medical wards: a controlled study. BMF 1985;290:9657.

28 Bien TH, Miller WR, Tonigan JS. Brief interventions for alcohol problems: a review. Addiction 1993;88:315-6. 